\title{
Geometrical aggregation of finite fuzzy sets
}

\author{
María Jesús Campión \\ Inarbe (Institute for Advanced Rersearch in Business and Economics) and Departamento de \\ Estadística, Matemáticas e Informática, Universidad Pública de Navarra, Pamplona (Spain) \\ Raquel G. Catalán \\ InaMat (Institute for Advanced Materials) and Departamento de Estadística, Matemáticas e \\ Informática, Universidad Pública de Navarra, Pamplona (Spain) \\ Esteban Induráin \\ InaMat (Institute for Advanced Materials) and Departamento de Estadística, Matemáticas e \\ Informática, Universidad Pública de Navarra, Pamplona (Spain) \\ Inmaculada Lizasoáin \\ InaMat (Institute for Advanced Materials) and Departamento de Estadística, Matemáticas e \\ Informática, Universidad Pública de Navarra, Pamplona (Spain) \\ Armajac Raventós-Pujol \\ Inarbe (Institute for Advanced Rersearch in Business and Economics) and Departamento de \\ Estadística, Matemáticas e Informática, Universidad Pública de Navarra, Pamplona (Spain)

\section{Óscar Valero} \\ Departament de Ciències Matemàtiques i Informàtica, Universitat de les Illes Balears, \\ Palma de Mallorca (Spain)
}

\begin{abstract}
A fuzzy set on a finite universe can be interpreted as a vector in a unit cube. This gives rise to a huge variety of approaches in order to aggregate finite fuzzy sets or to modify a given one. We analyze several geometrical methods and discuss possible applications in a multidisciplinary setting.

Keywords: Finite fuzzy sets; aggregation operators; Fermat points; Voronoi diagrams; ordered weighting averaging (OWA) aggregation operators.

Email addresses: mjesus.campion@unavarra.es (María Jesús Campión), raquel.garcia@unavarra.es (Raquel G. Catalán), steiner@unavarra.es (Esteban Induráin), ilizasoain@unavarra.es (Inmaculada Lizasoáin), armajac.raventos@unavarra.es (Armajac Raventós-Pujol), o.valero@uib.es (Óscar Valero)
\end{abstract}

Preprint submitted to International Journal of Approximate Reasoning 


\section{Introduction}

In fuzzy set theory it is typical to aggregate several fuzzy sets of a universe into a new fuzzy set, following some suitable criteria. Even when we are only dealing with one fuzzy set, it is also typical to modify it somewhere, according to some pre-established rules. When the fuzzy sets are framed on a finite universe with $n$ elements, those fuzzy sets can be considered as vectors in the $n$-dimensional unit cube $[0,1]^{n} \subset \mathbb{R}^{n}$. Therefore, any operation with those vectors can actually be understood as an aggregation operator of fuzzy sets of finite support. Among possible operations with vectors we may think of algebraic ones (e.g.: means), analytical ones (defined by means of suitable functions from the unit cube into the unit interval) and geometrical ones (as, for example, looking for the Fermat point of a triangle). Classical concepts in fuzzy set theory, as for instance triangular norms and conorms ([31]), copulas ([29]) and OWA operators ([53]) would also correspond to this general setting.

In the present paper we focus on geometrical methods.

Among possible applications we may think of different kinds of photo retouches in Image Processing, as well as aggregating individual opinions into a social one in natural mathematical contexts encountered in Decision Making, Social Choice and Mathematics of Finance (see e.g. [35]).

\section{Previous concepts}

First we introduce the classical concept of a fuzzy set of a universe.

\subsection{Fuzzy sets of a universe}

Throughout the paper, a set in the standard sense is said to be crisp, in opposition to the term "fuzzy" introduced below in Definition 1.

The standard definition of a fuzzy set goes as follows.

Definition 1. ([54, 7]) Let $U$ be a nonempty set, usually called a universe. A fuzzy set $X$ of $U$ is defined by means of a map $\mu_{X}: U \rightarrow[0,1]$. The map $\mu_{X}$ is said to be the membership function (or the indicator degree) of $X$.

The support of $X$ is the crisp subset $\operatorname{Supp}(X)=\left\{t \in U: \mu_{X}(t) \neq 0\right\} \subseteq U$, whereas the kernel of $X$ is the crisp subset $\operatorname{Ker}(X)=\left\{t \in U: \mu_{X}(t)=1\right\} \subseteq U$. The fuzzy subset $X$ is said to be normal provided that it has nonempty kernel.

Given $\alpha \in[0,1]$, the crisp subset of $U$ defined by $U_{\alpha}=\left\{t \in U: \mu_{X}(t) \geq \alpha\right\}$ is said to be the $\alpha$-cut of the fuzzy subset $X$.

Remark 1. Some authors use the nomenclature "fuzzy subset of a universe", instead of "fuzzy set". Observe in addition that through this Definition 1, the fuzzy set $X$ is indeed identified to its indicator function $\mu_{X}$. It is customary to use both notations, namely $X$ and $\mu_{X}$, interchangeably. 
Suppose that $U=\left\{u_{1}, \ldots, u_{n}\right\}$ is a finite universe ${ }^{1}$. We may identify a membership function $\mu_{X}: U \rightarrow[0,1]$ of a fuzzy set $X$ of $U$, with the vector $\left(x_{1}, \ldots, x_{n}\right)$ in the unit cube $[0,1]^{n}$. Here $\left.x_{i}=\mu_{X}\left(u_{i}\right) \quad i=1, \ldots, n\right)$.

In this setting, any operation with vectors in $[0,1]^{n}$ can immediately be interpreted as an operator that aggregates, into a new one, fuzzy sets of the given universe $U$.

\subsection{Aggregation and modification}

Let us discuss now a theoretical setting to understand well concepts as "aggregation" of fuzzy sets and "modification" of a fuzzy set.

Definition 2. In order to fuse $k$ fuzzy sets $\left\{X_{1}, \ldots, X_{k}\right\}$ on the same universe $U=\left\{u_{1}, \ldots, u_{n}\right\}$ we define an aggregation operator as a map $F: \mathcal{F}^{k} \rightarrow \mathcal{F}$, where $\mathcal{F}$ stands for the family of fuzzy sets of $U$. Thus, given $k$ membership functions $\left\{\mu_{X_{1}}, \ldots, \mu_{X_{k}}\right\}$ of fuzzy sets of $U, F\left(\mu_{X_{1}}, \ldots, \mu_{X_{k}}\right)$ will be the membership function of another fuzzy set, say $X_{F}$, of the universe $U$.

We may identify each membership function $\mu_{X_{i}} \quad(i=1, \ldots, k)$ to the $n$-dimensional vector $\bar{x}_{i}=\left(x_{i 1}, \ldots, x_{i n}\right)$ in the unit cube $[0,1]^{n}$, such that $\mu_{X_{i}}\left(u_{j}\right)=x_{i j} \quad(j=1, \ldots, n)$. In the same way, $\mu_{X_{F}}$ will be identified to the vector $\bar{x}_{F}=\left(x_{F 1}, \ldots, x_{F n}\right)$, with $\mu_{X_{F}}\left(u_{j}\right)=x_{F j}(j=1, \ldots, n)$.

Under this identification, we may write $F\left(\bar{x}_{1}, \ldots \bar{x}_{k}\right)=\bar{x}_{F}$.

In some typical questions related to fuzzy sets we just consider one fuzzy set, and we modify it someway. This generates the following definition.

Definition 3. Let $U=\left\{u_{1}, \ldots, u_{n}\right\}$ denote a finite universe. Let $\mathcal{F}$ stand for the family of fuzzy sets of $U$. A map $F: \mathcal{F} \rightarrow \mathcal{F}$ is said to be a modifier. Notice that the definition of a modifier is a particular case of the definition of aggregation operator, for the case in which $k=1$. However, the nuance is different here: if we only have one fuzzy set, we do not "aggregate". Instead, we modify. Observe also that after identifying $\mathcal{F}$ to $[0,1]^{n}$ a modifier becomes a function from the unit cube into itself. (For further information, see e.g. [36], pp. 110-117).

Example 1. A black and white digital picture can be understood as a function from a finite set of pixels (obviously, this will be the universe $U$ ) into the unit interval $[0,1]$. Each pixel $x \in U$ is assigned a number $u(x) \in[0,1]$ that measures the proportion of black ink on that pixel, so that $u(x)=0$ means that the pixel will appear as totally white, whereas $u(x)=1$ means that it will appear totally black, and any other number between 0 and 1 for $u(x)$ would correspond to an intensity of gray in the pixel $x$. In other words, a black and white picture

\footnotetext{
${ }^{1}$ Despite of considering a finite universe $U$ all along this paper (unless otherwise stated), in fact we could be working in a slightly more general context, namely we might assume that $U$ is any universe (finite or not) but the fuzzy sets considered on $U$ will always be of finite support.
} 
is a fuzzy set of the universe of pixels. Thus, in order to take a "negative" of a black and white picture, it is enough to revert the amounts of black and white at each pixel. And it is clear that the function $v: U \rightarrow[0,1]$ given by $v(x)=1-u(x) \quad(x \in U)$ corresponds to the picture in negative.

\subsection{Suitable properties that could be satisfied by aggregation operators}

In the search for aggregation operators it is usual to ask the operators we are interested in to accomplish some additional suitable properties that perhaps we could interpret as "common sense". By this reason we introduce now several definitions in that direction.

Definition 4. Following the notation of Definition 2, we say that the aggregation operator $F$ is fair provided that it satisfies the following conditions:

- i) (Unanimity) If $\bar{x}_{i}=\bar{x}$ for every $i \in\{1, \ldots, k\}$ then $F\left(\bar{x}_{1}, \ldots \bar{x}_{k}\right)=\bar{x}$.

- ii) (Anonimity) If $\sigma$ denotes any permutation of the set $\{1, \ldots, k\}$, then $F\left(\bar{x}_{1}, \ldots \bar{x}_{k}\right)=F\left(\bar{x}_{\sigma_{1}}, \ldots \bar{x}_{\sigma_{k}}\right)$.

- iii) (Continuity) $F$ is a continuous map from $\left([0,1]^{n}\right)^{k}$ into $[0,1]^{n}$, where on $[0,1]$ we consider the usual Euclidean topology and on $[0,1]^{n}$ as well as on $\left([0,1]^{n}\right)^{k}$ we consider the corresponding product topologies.

Remark 2. This kind of aggregators, namely those that satisfy the aforementioned properties of unanimity, anonimity and continuity are typical in Mathematical Social Choice, in the study of the so-called Chichilnisky's topological rules of aggregation of preferences, see e.g. [18] for further details. As a matter of fact, this sort of aggregation operators had already appeared much earlier in theoretical studies of Geometry and Topology, see e.g. [4].

From a computational point of view, having in mind the possibility of aggregating fuzzy sets by means of some computer program, it is clear that in order to aggregate $k$ fuzzy sets, we may use some suitable algorithm. Hence, it seems more practical to have at hand algorithms that act on two sets and can be iterated rather than other ones that need to consider a priori all the $k$ fuzzy sets to be fused. This inspires the next definition.

Definition 5. Consider a natural number $k>2$. An aggregation operator $F: \mathcal{F}^{k} \rightarrow \mathcal{F}$ is said to be reducible if there exists a bivariate operator $G$ : $\mathcal{F}^{2} \rightarrow \mathcal{F}$ such that for every $\left(X_{1}, \ldots, X_{k}\right) \in \mathcal{F}^{k}$ it holds that $F\left(X_{1}, \ldots, X_{k}\right)=$ $G\left(G\left(\ldots\left(G\left(X_{1}, X_{2}\right), \ldots\right), X_{k-1}\right), X_{k}\right){ }^{2}$

Even in case of iterating someway a given bivariate aggregation operator $F$ : $\mathcal{F}^{2} \rightarrow \mathcal{F}$, the resulting fuzzy set may depend on the way we proceed. In general, given $X, Y, Z \in \mathcal{F}$, it may happen that $F(F(X, Y), Z) \neq F(X, F(Y, Z))$. By this reason, we introduce the next definition.

\footnotetext{
${ }^{2}$ That is, if $k=3$ then $F\left(X_{1}, X_{2}, X_{3}\right)=G\left(G\left(X_{1}, X_{2}\right), X_{3}\right) . \quad$ If $k=4$, then $F\left(X_{1}, X_{2}, X_{3}, X_{4}\right)=G\left(G\left(G\left(X_{1}, X_{2}\right), X_{3}\right), X_{4}\right)$, and so on.
} 
Definition 6. A bivariate aggregation operator $F: \mathcal{F}^{2} \rightarrow \mathcal{F}$ is said to be associative if $F(F(X, Y), Z)=F(X, F(Y, Z))$ holds for every $X, Y, Z \in \mathcal{F}$.

Remark 3. Notice that by systematically iterating an associative bivariate aggregation operator $F$, we may indeed fuse $k$ fuzzy sets of the universe $U$, for any $k \geq 2$.

Among aggregation operators on fuzzy sets on a finite universe, and taking into account the identification to those fuzzy sets and vectors in the unit cube $[0,1]^{n}$ we may consider those operators that "act coordinatewise", in the sense of the next definition.

Definition 7. A map $F:\left([0,1]^{n}\right)^{k} \rightarrow[0,1]^{n}$ is said to be coordinatewise realizable if there exists a function $f:[0,1]^{k} \rightarrow[0,1]$ such that if $\bar{x}_{i}=\left(x_{i 1}, \ldots, x_{i n}\right)(i=$ $1, \ldots, k)$ and $\bar{x}_{F}=F\left(\bar{x}_{1}, \ldots \bar{x}_{k}\right)=\left(x_{F 1}, \ldots, x_{F n}\right)$, then it holds true that $x_{F j}=f\left(x_{1 j}, \ldots, x_{k j}\right) \quad(j=1, \ldots, n)$.

Remark 4. The search for aggregation operators that satisfy some of the definitions introduced above (namely, fairness, reducibility, associativity and/or coordinatewise realizability) is clearly related to the solution of functional equations on several variables. (See e.g. $[14,13,22,16]$ ).

\subsection{A variety of methods to aggregate finite fuzzy sets}

Any method to aggregate fuzzy sets on a finite universe $U=\left\{u_{1}, \ldots, u_{n}\right\}$ tries to build and describe maps from $\left([0,1]^{n}\right)^{k}$ into $[0,1]^{n}$. However, to do so, many different approaches may appear. Among them, a first classification could be: i) algebraic methods, ii) analytical methods, iii) geometrical methods.

Needless to say, this first attempt to classify the different methods is by no means exclusive. That is, some methods share different sort of techniques, being, for instance, analytical in some aspects and algebraic or geometrical in other features.

Let us briefly discuss here what can be encountered in this setting:

First we may consider typical operations with vectors in $[0,1]^{n}$ seen as a subset of the real vector space $\mathbb{R}^{n}$. Thus, given $k$ vectors $\bar{x}_{1}, \ldots, \bar{x}_{k} \in[0,1]^{n}$, its arithmetic mean $\frac{\bar{x}_{1}+\ldots+\bar{x}_{k}}{k}$ is well defined and actually takes values in $[0,1]^{n}$. Moreover, it is coordinatewise realizable. When $k=2$, the mean $M$ given by $M\left(\bar{x}_{1}, \bar{x}_{2}\right)=\frac{\bar{x}_{1}+\bar{x}_{2}}{2}$ is not associative. Notice also that $\frac{1}{k} \cdot\left(\bar{x}_{1}+\ldots+\bar{x}_{k}\right)=$ $\frac{2}{k-1} \cdot\left(\sum_{i<j, 1<i, j \leq k} M\left(\bar{x}_{i}, \bar{x}_{j}\right)\right)$, so the arithmetic mean for $k$ vectors can still be expressed someway in terms of several arithmetic means that only involve two vectors each. (Compare this fact to the, more restrictive, Definition 5).

Of course, many other different means can be considered in this setting. For instance, taking into account that $t \in[0,1] \Longrightarrow t^{\frac{1}{k}} \in[0,1]$ holds true for every $t$ in the unit interval, we may consider the function $f:[0,1]^{k} \rightarrow[0,1]$ given by $f\left(t_{1}, \ldots t_{k}\right)=\left(t_{1} \cdot \ldots \cdot t_{k}\right)^{\frac{1}{k}}$, usually known as the geometrical mean. Then, through $f$ and acting coordinatewise we can build an aggregation operator from $\left([0,1]^{n}\right)^{k}$ into $[0,1]^{n}$, that will be obviously coordinatewise realizable in the sense of Definition 7. For further details, see e.g. [15]. 
The search for analytical expressions that give rise to suitable aggregators is typical in this literature. For instance, well-behaved associative copulas and their generalizations (see e.g. [44]), as well as triangular norms and conorms have been widely analyzed in these contexts from many points of view. (See e.g. [3]). Natural questions that appear here are related to the solution of some functional equations. (See e.g., [12, 13, 14, 21, 33]).

In addition, other analytical, combinatorial or algebraic methods can also be applied to compare two different fuzzy sets. To put an easy example, given two vectors $\bar{x}, \bar{y}$ in the unit cube $[0,1]^{n}$ we could pay attention to the number of coordinates that differ, in order to give a naive idea about how disparate are those vectors (or fuzzy sets of a finite universe). Another easy idea is to measure someway (e.g., through an adequate norm in $\mathbb{R}^{n}$ ) the vector $\bar{x}-\bar{y}$.

\section{Geometrical methods to aggregate finite fuzzy sets}

Geometrical methods can also be considered at this stage. In this Section 3 we focus on this approach, namely the construction of aggregation operators by means of some suitable geometrical idea. As far as we know, these methods are not so common (nor well-known, either) in the fuzzy set theory literature.

\subsection{Some triangle centers}

To put a motivating example, suppose that $U=\left\{u_{1}, u_{2}\right\}$ is the universe. Since $n=2$, the family of fuzzy sets of $U$ can be identified to the unit square $[0,1]^{2}$. Now suppose that we have at hand three fuzzy sets of $U$ to be aggregated some way. Identifying the corresponding fuzzy sets to points in the unit square, we will have three points in $[0,1]^{2}$, say $A=\left(x_{1}, x_{2}\right), B=\left(y_{1}, y_{2}\right)$ and $C=$ $\left(z_{1}, z_{2}\right)$. Obviously, these points define a triangle in the unit square. Given those three points we may use geometrical ideas to find another one that aggregates them. To do so, first we may think of various typical triangle centers (see e.g. [11]).

Suppose that the points $A, B$ and $C$ are not collinear, so that they are the vertices of a non degenerated triangle.

i) The circumcenter of the triangle can be considered a good aggregation, because it is equally distant from $A, B$ and $C$.

ii) The barycenter (or center of gravity, or centroid) is also a suitable aggregation. Its coordinates $(a, b)$ are obtained through the arithmetic mean, that is $(a, b)=\left(\frac{x_{1}+y_{1}+z_{1}}{3}, \frac{x_{2}+y_{2}+z_{2}}{3}\right)$. Notice, in addition, that this kind of aggregation is fair and coordinatewise realizable in the sense of Definitions 4 and 7.

iii) The Fermat point (also known as the Fermat-Torricelli point) $F$ is defined as the point $F$ such that the sum of the distances of $F$ to the vertices $A$, $B$ and $C$ is kept to a minimum, see e.g. [19, 30, 32, 39, 34]. 
Remark 5. Among these aforementioned three triangle centers, the circumcenter only makes sense if $A, B$ and $C$ do not lie in the same straight line. Instead, the barycenter, understood as the point $\left(\frac{x_{1}+x_{2}+x_{3}}{3}, \frac{y_{1}+y_{2}+y_{3}}{3}\right)$ is always well-defined. Also, it is straightforward to see that the given three collinear points $A, B, C$, its median $F$ minimizes the sum of distances to $A, B, C$. Thus, in the case of collinear points we may define the Fermat point as the median (see [6] for a further account). Perhaps by this reason, Fermat-Torricelli points and their generalizations are also called geometric medians in this literature.

Definition 8. The geometric median or Fermat point of a set of $k$ sample points in the Euclidean space $\mathbb{R}^{n}$ is the point that minimizes the sum of distances to those given points.

Proposition 1. Consider a set of $k \geq 3$ sample points in $\mathbb{R}^{n}$. If those points are not collinear, then its Fermat point is unique.

Proof: See [47].

Remark 6. It is clear that the Fermat point of a set of $k$ sample points in $\mathbb{R}^{n}$ only depends on the set as a whole, and not on the order in which those points appear in it. That is, the aggregation operator that assigns the Fermat point to a set is anonymous (see Definition 4). Moreover, it is also unanimous. Concerning its continuity, a good study appears in [39] for the particular case $k=3$, since it can be seen there how the coordinates of the Fermat point continuously depend on the coordinates of the three given sample points. The analytical expression of those coordinates, even lengthy and tedious to be obtained, has explicitly been got and discussed in that paper [39]. Therefore, for $k=3$, the aggregation operator that assigns the geometric median to a given set of three points is fair in the sense of Definition 4. However, when $k>3$ a trouble can appear because the Fermat point could fail to be unique in certain cases that involve collinear sample points.

With respect to the circumcenter of a triangle of vertices $A, B$ and $C$, the aggregation operator that assigns the circumcenter to that set of three sample points (namely, the vertices) is anonymous. It is also unanimous if we understand that when the three vertices of a triangle tend to a point, the circumcenter of such triangle also tends to that point. As regards the continuity, a trouble appears because when the three points $A, B, C$ are collinear, so that the corresponding triangle that they would define degenerates to a segment, no circumcenter can be defined. Moreover, if $A=\left(x_{1}, x_{2}\right) ; B=\left(y_{1}, y_{2}\right) ; C=\left(z_{1}, z_{2}\right)$ are the vertices of a triangle, we know that the circumcenter is a point $P=(a, b)$ that is equidistant from $A, B$ and $C$. This fact can not be interpreted coordinatewise, for $a$ is not, in general, a number such that $\left|a-x_{1}\right|=\left|a-y_{1}\right|=\left|a-z_{1}\right|$. Neither $b$ satisfies, in general, that $\left|b-x_{2}\right|=\left|b-y_{2}\right|=\left|b-z_{2}\right|$. In other words, the aggregation operator that assigns the circumcenter to the vertices of a triangle fails to be coordinatewise realizable, in the sense of Definition 7.

At this stage, we may notice that things are really different whenever we have more than three points in the unit square. Concerning an analogue to the 
circumcenter, the insurmountable trouble is that four or more points can fail to be cocyclic, so that there is no point that is equally distant to them all. The analogue to the barycenter, however, continues being an excellent aggregation, since the arithmetic mean is always well defined in the unit cube, disregarding its dimension. In particular, it is well defined in the unit square. Finally, for more than three points the analogous of the Fermat-Torricelli point is always well-defined, too. Since the sum of distances constitutes a continuous function on the unit square, by Weierstrass's theorem that function attains its minimum on $[0,1]^{2}$ (see e.g. [34]).

\subsection{Fermat and Weber points}

It is clear that the Fermat point of a triangle is a particular case of a triangle center. The problem of finding the Fermat point can be generalized in several ways: we may consider ab initio a number of points different from three, and then look for points that minimize the sume of distances to those given points. Also, we can add weight to each of the points and look for points that minimize weighted distances, usually called Weber points (see e.g. [50, 52, 51, 17]). Moreover, we can work in more than two dimensions (see e.g. [1]).

Definition 9. Let $\left\{P_{1}, \ldots, P_{k}\right\}$ be a set of $k$ sample points in the Euclidean space $\mathbb{R}^{n}$. Let $W=\left\{\omega_{1}, \ldots, \omega_{k}\right\}$ denote a set of non-negative real numbers, called weights. We say that a point $P$ in $\mathbb{R}^{n}$ is the Weber point as regards $W$, if it minimizes the expression $\sum_{i=1}^{k} \omega_{i} . d\left(P_{i}, P\right)$, where $d\left(P_{i}, P\right)$ stands for the Euclidean distance between the points $P_{i}$ and $P(i=1, \ldots, k)$.

Remark 7. It is straightforward to see that if all the weights $\left\{\omega_{1}, \ldots, \omega_{k}\right\}$ are non-negative rational numbers, so that $w_{i}=\frac{p_{i}}{q_{i}}(1 \leq i \leq k)$, then the Weber point as regards $W$ can be understood as the Fermat point of a new set of sample points where each $P_{i}$ is repeated $M \cdot \frac{p_{i}}{q_{i}}$ times, with $M=q_{1} \cdot \ldots \cdot q_{k}$.

Since distances are continuous functions with respect to the usual Euclidean topology in the real space, Fermat points as well as Weber points (or its analogous in other dimensions) always exist by the well-known Weierstrass's theorem in Calculus, because the unit square (as well as any unit cube) is compact with respect to the usual topology. However, a difficulty may still appear, since Weierstrass's theorem does not guarantee the uniqueness of a minimum point of a continuous function on a compact set. To put an evident example, consider the unit square and just two points $\left(x_{1}, x_{2}\right)$ and $\left(y_{1}, y_{2}\right)$ there. Then notice that any point $(a, b)$ that lies in the segment joining $\left(x_{1}, x_{2}\right)$ and $\left(y_{1}, y_{2}\right)$ is a Fermat point in the sense that it minimizes the sum of distances to those two points. Indeed, that sum of distances is the same for every point in that segment.

Remark 8. The Fermat point of a triangle does not coincide, in general, with the barycenter. Indeed, if the triangle of vertices $A, B, C$ has an angle bigger than or equal to $\frac{2}{3} \pi$ radians (120 sexagesimal degrees), the vertex corresponding to that angle is the Fermat point. Otherwise, when each of the three angles is smaller that $\frac{2}{3} \pi$ radians, the Fermat point is obtained by constructing outer 
equilateral triangles on each side of the triangle of vertices $A, B, C$. Constructing these equilateral triangles introduces three new vertices, say $P, Q$ and $R$. Draw a line from each of these new vertices to the vertex (of the main triangle of vertices $A, B, C$ ) that lies directly opposite to them, for example: from $A$ to $R$. It is well-known then that these three lines meet in a point $F$, that is actually the Fermat point of the given triangle. (The result is clearly related to Napoleon's theorem in Classical Geometry, see e.g. [20]).

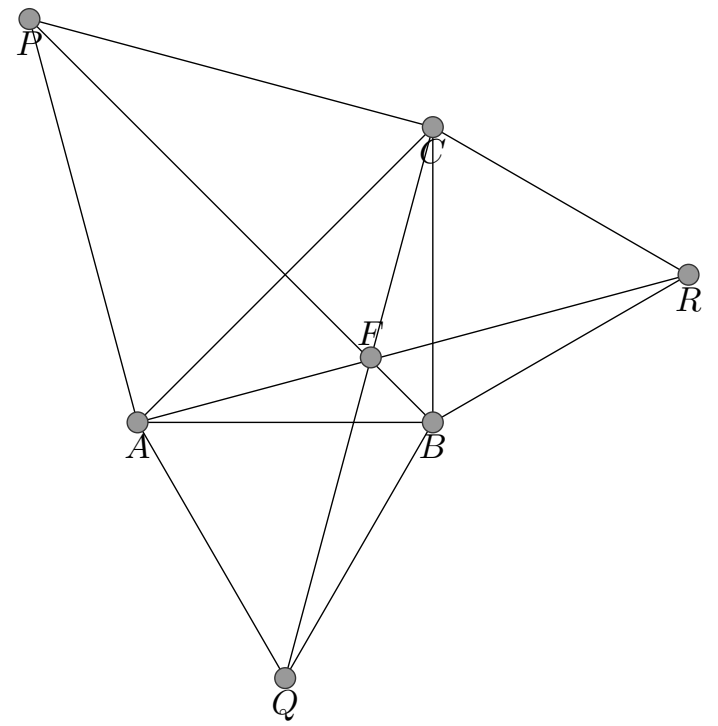

By the way, the computation of Fermat points is not a coordinatewise realizable aggregation operator (see Definition 7 ). To put an example, consider the points $A=(0,0) ; B=(1,0) ; C=(1,1)$ in the unit plane. The median of their first coordinates is 1 , whereas the median of the second ones is 0 . However, $(1,0)$ is not the geometric median (or Fermat point) of that triangle. Here it is straightforward to see that the Fermat point lies inside the triangle and does not coincide with any vertex.

Also, it is well known that given four coplanar points, if one of those points lies inside the triangle formed by the other three points, then the Fermat point (or geometric median) is that point. Otherwise, the four points form a convex quadrilateral and the Fermat point is the intersection point of the diagonals of such quadrilateral (see [40]).

In addition, if we try to compute the Fermat-Torricelli point of a triangle just by using analytical methods, the process is lengthy, difficult and discouraging, as analyzed in depth in [39]. Thus, given three points $A=\left(x_{1}, x_{2}\right), B=\left(y_{1}, y_{2}\right)$ and $C=\left(z_{1}, z_{2}\right)$ in the unit square, we want to find another point $F=(a, b)$ such that

$$
\sqrt{\left(x_{1}-a\right)^{2}+\left(x_{2}-b\right)^{2}}+\sqrt{\left(y_{1}-a\right)^{2}+\left(y_{2}-b\right)^{2}}+\sqrt{\left(z_{1}-a\right)^{2}+\left(z_{2}-b\right)^{2}}
$$


is a minimum. Here, unlike the case of minimization of square distances, that is very easy to handle and typically arises in Statistics (and in this case would correspond to finding the minimum value ${ }^{3}$ of $\left[\left(x_{1}-a\right)^{2}+\left(x_{2}-b\right)^{2}+\left(y_{1}-a\right)^{2}+\right.$ $\left.\left(y_{2}-b\right)^{2}+\left(z_{1}-a\right)^{2}+\left(z_{2}-b\right)^{2}\right]$, the analytical study of the existence of the Fermat point becomes crazy. This is because the square roots complicate the operations beyond reasonable limits.

In this line, unfortunately, when more than three points are involved no general direct geometrical method is known to find a Fermat point. And, needless to say, the analytical study becomes even crazier.

Nevertheless, there is a recursive algorithm that calculates approximations to the Fermat point. To describe it, we introduce the next Definition 10.

Definition 10. Let $X=\left\{\bar{x}_{i}: 1 \leq i \leq k\right\}$ be a set of not collinear $k$ vectors (or sample points) in the Euclidean space $\mathbb{R}^{n}$. Given a vector $\bar{y} \in \mathbb{R}^{n}$, we say that $\bar{y}$ generates a Weiszfeld sequence if taking $\bar{y}_{0}=\bar{y}$ and, recurrently,

$$
\bar{y}_{j+1}=\frac{\sum_{i=1}^{k} \frac{\bar{x}_{i}}{\left\|\bar{x}_{i}-\bar{y}_{j}\right\|}}{\sum_{i=1}^{k} \frac{1}{\left\|\bar{x}_{i}-\bar{y}_{j}\right\|}},
$$

it happens that $\bar{y}_{j}$ does not belong to $X$ for any $j \in \mathbb{N}$.

(Here, given a vector $\bar{z}=\left(z_{1}, \ldots, z_{n}\right) \in \mathbb{R}^{n}$, we denote by $\|\bar{z}\|$ its Euclidean norm. That is: $\left.\|\bar{z}\|=\sqrt{z_{1}^{2}+\ldots+z_{n}^{2}}\right)$.

The procedure of obtaining the sequence $\left(\bar{y}_{j}\right)_{j \in \mathbb{N}}$ this way is known as the Weiszfeld's algorithm (see [51]).

Proposition 2. The Weiszfeld's algorithm converges for almost all vectors ${ }^{4}$ $\bar{y} \in \mathbb{R}^{n}$. In addition, the algorithm can be modified in a suitable way that allows us to handle all possible initial points, giving rise to a sequence that converges to the Fermat point.

PROOF: See [47] for a proof and further details.

This geometrical approach can also be used to give an idea of dispersion among a family of different fuzzy sets of a universe. Thus, we may compute the Fermat point corresponding to that family, and the corresponding minimum of the sum of distances to the Fermat point of each given fuzzy set (understood as a vector in the unit cube).

\subsection{Ordered weighted aggregations and other geometrical coordinatewise realiz- able operators}

As commented before, the aggregation operator that computes a Fermat point is not coordinatewise realizable, in general. Some other typical operators,

\footnotetext{
${ }^{3}$ It is straightforward to see that such minimum appears at the barycenter $\left(\frac{x_{1}+y_{1}+z_{1}}{3}, \frac{x_{2}+y_{2}+z_{2}}{3}\right)$ of the triangle of vertices $A, B, C$.

${ }^{4}$ Obviously, the algorithm does not work when when one of its estimates falls on one of the given points in the set $V$ of sample points.
} 
frequently used in fuzzy set theory, are built so that they are coordinatewise realizable in the sense of Definition 7 . That is, they are constructed in a way such that the $j$-th coordinate of the vector that appears as the final result of the aggregation made only depends from the $j$-th coordinates of the vectors to be aggregated.

Unfortunately, some of them do not correspond to a clear geometrical intuition. That is, they are constructed by means of some analytical expression, that sometimes is hard to be geometrically visualized. Anyway, some of them (e.g.: centroids, barycenters and arithmetic means again) still correspond to geometrical ideas. Moreover, other ones as the OWA operators ([53]), admit some geometrical interpretation, and are of capital importance in applications (e.g. in fuzzy set theory).

To introduce here a short list of aggregation operators that share this feature (see $[5,53])$, first we introduce a few definitions.

Definition 11. Given a vector $\bar{u}=\left(u_{1}, \ldots, u_{k}\right) \in[0,1]^{k}$, its non-increasingly rearranged vector is $R(\bar{u})=\left(u_{(1)}, \ldots, u_{(k)}\right)$, where the coordinates of $\bar{u}$ have now been rearranged in non-increasing order, that is $u_{(1)} \geq \ldots \geq u_{(k)}$.

Definition 12. Given a set $W=\left\{\omega_{1}, \ldots, \omega_{k}\right\}$ of non-negative real numbers such that, in addition, $\omega_{1}+\ldots+\omega_{k}=1$, (also known as weights), the ordered weighting averaging function (OWA function) relative to $W$ (see [53]) is the function $\Phi_{W}:[0,1]^{k} \rightarrow[0,+\infty)$ given by

$$
\Phi_{W}(\bar{u})=\sum_{j=1}^{k} \omega_{j} \bar{u}_{(j)} .
$$

Now we introduce a few coordinatewise realizable operators:

Let $X=\left\{\bar{x}_{i}=\left(x_{i 1}, x_{i 2}, \ldots, x_{i n}\right): 1 \leq i \leq k\right\}$ be a cloud of $k$ vectors in the unit cube $[0,1]^{n}$. If we pay attention only to the $j$-component $(1 \leq j \leq n)$ of the vectors $\bar{x}_{i} \quad(1 \leq i \leq k)$, once $j$ has been fixed we get a column vector, namely:

$$
v^{j}=\left(\begin{array}{l}
x_{1 j} \\
x_{2 j} \\
\cdots \\
x_{k j}
\end{array}\right)
$$

Let $R\left(v^{j}\right)$ stand for the non-increasingly rearranged vector associated to $v^{j}$, that is: $R\left(v^{j}\right)=\left(\begin{array}{l}x_{(1) j} \\ x_{(2) j} \\ \ldots \\ x_{(k) j}\end{array}\right)$, so that now $x_{(1) j} \geq x_{(2) j} \geq \ldots \geq x_{(k) j}$.

i) The arithmetic mean looks for a vector $\bar{y}=\left(y_{1}, \ldots, y_{n}\right)$ such that for every 
$1 \leq j \leq n$, the coordinate $y_{j}$ minimizes the sum

$$
\sum_{i=1}^{k}\left(x_{i j}-y_{j}\right)^{2}
$$

Notice that this happens if and only if $\bar{y}$ minimizes the sum of the squares of the distances to the ponits in the cloud $X$, that is

$$
\sum_{i=1}^{k}\left[d\left(\bar{x}_{i}, \bar{y}\right)\right]^{2}=\sum_{j=1}^{n} \sum_{i=1}^{k}\left(x_{i j}-y_{j}\right)^{2} .
$$

(Here $d$ stands for the Euclidean distance in the plane $\mathbb{R}^{2}$ ).

It is well-known that in this case the solution is the analogous to the barycenter or centroid of a triangle. That is $y_{j}=\frac{x_{1 j}+\ldots+x_{k j}}{k}$ holds true for every $1 \leq j \leq n$.

ii) The weighted arithmetic mean looks for a vector $\bar{y}=\left(y_{1}, \ldots, y_{n}\right)$ such that for every $1 \leq j \leq n$, the coordinate $y_{j}$ minimizes the sum

$$
\sum_{i=1}^{k} \omega_{i}\left(x_{i j}-y_{j}\right)^{2}
$$

iii) The ordered weighting averaging aggregation operator (OWA operator) ([53]) looks for a vector $\bar{y}=\left(y_{1}, \ldots, y_{n}\right)$ such that for every $1 \leq j \leq n$, the coordinate $y_{j}$ minimizes the sum

$$
\sum_{i=1}^{k} \omega_{i}\left(x_{(i) j}-y_{j}\right)^{2} .
$$

iv) The weighted median looks for a vector $\bar{y}=\left(y_{1}, \ldots, y_{n}\right)$ such that for every $1 \leq j \leq n$, the coordinate $y_{j}$ minimizes the sum

$$
\sum_{i=1}^{k} \omega_{i}\left|x_{i j}-y_{j}\right| .
$$

v) The ordered weighted median looks for a vector $\bar{y}=\left(y_{1}, \ldots, y_{n}\right)$ such that for every $1 \leq j \leq n$, the coordinate $y_{j}$ minimizes the sum

$$
\sum_{i=1}^{k} \omega_{i}\left|x_{(i) j}-y_{j}\right| .
$$

(Here $\left\{\omega_{1}, \ldots, \omega_{k}\right\}$ is a set of non-negative real numbers with $\omega_{1}+\ldots+\omega_{k}=1$. These $\omega_{i}(1 \leq i \leq k)$ are said to be the weights. And $x_{(i) j}$ is the $i$-th coordinate of $R\left(v^{j}\right)$, the non-increasing rearranged vector associated to $\left.v^{j} ; 1 \leq j \leq n\right)$. 
Remark 9. The OWA coordinatewise procedure aggregates the $k$ original vectors in the cloud $X=\left\{\bar{x}_{i}=\left(x_{i 1}, x_{i 2}, \ldots, x_{i n}\right): 1 \leq i \leq k\right\}$ so that the resulting aggregated point is the vector $\left(\Phi_{W}\left(x_{11}, x_{21}, \ldots, x_{k 1}\right), \ldots, \Phi_{W}\left(x_{1 n}, x_{2 n}, \ldots, x_{k n}\right)\right)$.

Geometrically, the rearrangement through $\Phi_{W}$ of each column vector $v^{j}=$ $\left(\begin{array}{l}x_{1 j} \\ x_{2 j} \\ \cdots \\ x_{k j}\end{array}\right)$

$(1 \leq j \leq n)$ has the effect of replacing the original "row" vectors in

the cloud, namely $X=\left\{\bar{x}_{i}=\left(x_{i 1}, x_{i 2}, \ldots, x_{i n}\right): 1 \leq i \leq k\right\}$, by a new set $X^{\prime}$ of $k$ vectors in $[0,1]^{n}$, defined as follows:

$$
X^{\prime}=\left\{\bar{x}_{1}^{\prime}=\left(x_{(1) 1}, x_{(1) 2}, \ldots, x_{(1) n}\right), \ldots, \bar{x}_{k}^{\prime}=\left(x_{(k) 1}, x_{(k) 2}, \ldots, x_{(k) n}\right)\right\},
$$

and now satisfying that:

$x_{(1) 1} \geq \ldots \geq x_{(k) 1} ; \ldots ; x_{(1) n} \geq \ldots \geq x_{(k) n}$.

These new points lie on an ascending curve in the unit cube $[0,1]^{n}$. Notice that if we aggregate now the points in $X^{\prime}$ by means of a weighted arithmetic mean with the weights given by $W$, we obtain again the vector

$$
\left(\Phi_{W}\left(x_{11}, x_{21}, \ldots, x_{k 1}\right), \ldots, \Phi_{W}\left(x_{1 n}, x_{2 n}, \ldots, x_{k n}\right)\right)
$$

as the resulting point of the aggregation. Observe also that if the weights are $\{1,0, \ldots, 0\}$ we obtain the vector corresponding to a "maximum" among the vectors in $X^{\prime}$, so that each of its coordinates, say the $j$-th, takes the maximum value $\max _{1 \leq i \leq n} x_{i j}$. Similarly, if the weights are $\{0, \ldots, 0,1\}$ we obtain the vector corresponding to a "minimum" among the vectors in $X^{\prime}$.

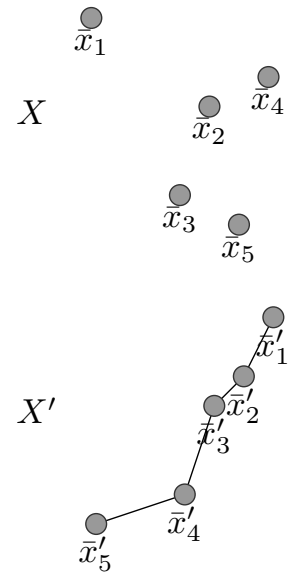

(See e.g. [37] for a further account).

\subsection{Voronoi diagrams and regions}

Suppose that a finite number of points $\left\{\bar{x}_{1}, \ldots, \bar{x}_{k}\right\}$ are located in a bounded region $\mathcal{R}$ of the plane. Now, make a partition of the region $\mathcal{R}$ in non-overlapping subregions $\mathcal{R}_{1}, \ldots \mathcal{R}_{k}$, such that $x_{i}$ lies in $\mathcal{R}_{i}\left(1 \leq i \leq k\right.$ and, in a sense, $\mathcal{R}_{i}$ 
represents the subregion of $\mathcal{R}$ that is controlled or dominated by $x_{i}$. How could we understand this idea with mathematical rigor?

Let us introduce some definition to settle better this problem.

Definition 13. Let $d$ stand for the Euclidean distance in the plane $\mathbb{R}^{2}$. Given a region $\mathcal{R}$ in $\mathbb{R}^{2}$, and $k$ points $\bar{x}_{1}, \ldots, \bar{x}_{k} \in \mathcal{R}$ we call Voronoi polygon or Voronoi cell (see $[48,49]) \mathcal{R}_{i}$ associated to the point $\bar{x}_{i}(1 \leq i \leq k)$ to the region

$$
\mathcal{R}_{i}=\left\{\bar{x} \in \mathcal{R}: d\left(\bar{x}, \bar{x}_{i}\right)=\min _{1 \leq j \leq k} d\left(\bar{x}, \bar{x}_{j}\right)\right\} .
$$

If $i \neq j$, then $\mathcal{R}_{i} \cap \mathcal{R}_{j}=\left\{x \in \mathcal{R}: d\left(\bar{x}, \bar{x}_{i}\right)=d\left(\bar{x}, \bar{x}_{j}\right)=\min _{1 \leq l \leq k} d\left(\bar{x}, \bar{x}_{l}\right)\right\}$. Moreover, the regions $\mathcal{R}_{i}$ and $\mathcal{R}_{j}$ share no interior point.

Furthermore,

$$
\bigcup_{1 \leq i \leq k} \mathcal{R}_{i}=\mathcal{R} .
$$

Remark 10. Voronoi polygons provide a partition of the region $\mathcal{R}$. Such partition, also known today as the Voronoi diagram, had already been implicitly considered by R. Descartes much earlier, in 1644 (see [24]). It was also used by P.G.L Dirichlet in 1850 (see [25]). These diagrams were extended to more dimensions by Voronoi in 1907-1908 (see $[48,49]$ ). Since then, the regions $\mathcal{R}_{i}$ are given the name of Voronoi cells. These diagrams can be found in nature: giraffe fur, turtle shells or fields of drying mud follow this pattern.

Independently from, and almost contemporarily to Voronoi's work, A.H. Thiessen introduced in 1911 the same idea (see [46]) in studies concerning weather (e.g.: regions where rain is more probable). By this reason, in some texts Voronoi's cells are named Thiessen's cells or Voronoi-Thiessen cells. (See also $[28,38,9]$ for a further account).

Observe that given $\bar{x}_{i} \neq \bar{x}_{j} \in X$, if we build the bisector of the segment that joins $\bar{x}_{i}$ to $\bar{x}_{j}$, and denote $H\left(\bar{x}_{i}, \bar{x}_{j}\right)$ the resulting half-plane in $\mathbb{R}^{2}$ to which $\bar{x}_{i}$ belongs, then $\mathcal{R}_{i}=\mathcal{R} \cap\left[\bigcap_{j \neq i} H\left(\bar{x}_{i}, \bar{x}_{j}\right)\right]$. Thus we see that the Voronoi diagram of a set of $k$ points in a region $\mathcal{R}$ is got by intersecting the Voronoi diagram of the same set of points in the whole plane $\mathbb{R}^{2}$ with the given region $\mathcal{R}$.

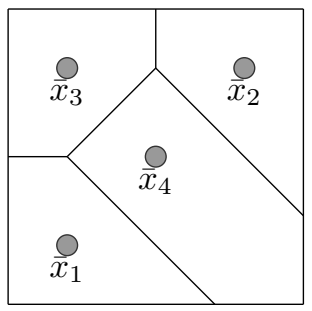

Henceforward in this subsection 3.4, unless otherwise stated, we will only consider here Voronoi diagrams of sets of points in the whole plane $\mathbb{R}^{2}$. 
Proposition 3. Let $P=\left\{\bar{x}_{1}, \ldots, \bar{x}_{k}\right\}$ denote a set of $k$ points in the real plane $\mathbb{R}^{2}$. Let $V_{P}=\left\{\mathcal{R}_{i} ; 1 \leq i \leq n\right\}$ denote the Voronoi diagram associated to $P$ in the whole plane $\mathbb{R}^{2}$. The following properties hold true:

i) Every Voronoi cell $\mathcal{R}_{i}$ is convex.

ii) A Voronoi cell $\mathcal{R}_{i}$ is unbounded if and only if $\bar{x}_{i}$ belongs to the boundary of the convex hull of $P$.

iii) All the boundaries of the regions $\mathcal{R}_{i}$ are whole straight lines (indeed parallel one another) if and only if the set $P$ is collinear.

iv) If $i \neq j$, the boundary of $\mathcal{R}_{i} \cap \mathcal{R}_{j}$ is a half-line if and only if $P$ is not collinear and $\bar{x}_{i}, \bar{x}_{j}$ are consecutive points in the boundary of the convex hull of $P$.

v) If $i \neq j$, The boundary of $\mathcal{R}_{i} \cap \mathcal{R}_{j}$ is a segment if and only if $P$ is not collinear and $\bar{x}_{i}$ or $\bar{x}_{j}$ belongs to the interior of the convex hull of $P$.

Proof: Just notice that given $1 \leq i \leq n$ the region $\mathcal{R}_{i}$ appears as $\mathcal{R}_{i}=$ $\cap_{j \neq i} H\left(\bar{x}_{i}, \bar{x}_{j}\right)$, an intersection of half-planes. Hence, the Voronoi cells are convex, and a region $\mathcal{R}_{i}$ unbounded only if $\bar{x}_{i}$ is in the boundary of the convex hull of $P$. This proves i) and ii). (See also $[41,38,9]$ for further details).

In order to prove iii), first we consider that the set $P$ only consist of two points, that is: $P=\left\{x_{1}, x_{2}\right\}$. Then clearly the Voronoi diagram has only two cells, any of them is a half-plane, and the only boundary in $V_{P}$ is the bisector to the segment that joins $\bar{x}_{1}$ to $\bar{x}_{2}$. If $P$ has more than two points, then if $P$ is collinear, given three consecutive points $\bar{x}_{i-1}, \bar{x}_{i}, \bar{x}_{i+1}$, the bisectors of the segments that join $\bar{x}_{i-1}$ to $\bar{x}_{i}$ and $\bar{x}_{i}$ to $\bar{x}_{i+1}$ are parallel (whole) straight lines and they constitute the boundaries between the cells of those consecutive points. If three points $\bar{x}_{i-1}, \bar{x}_{i}, \bar{x}_{i+1}$ in $P$ are not collinear then the bisectors of the segments that join $\bar{x}_{i-1}$ to $\bar{x}_{i}$ and $\bar{x}_{i}$ to $\bar{x}_{i+1}$ are no longer parallel. Therefore the boundaries cannot be whole straight lines. One of these boundaries is a half-line if the cells are unbounded, which happens only if the points are in the boundary of the convex hull of $P$. Otherwise the boundary between two cells is a segment. (See also Theorem 7.2 in [9]).

Definition 14. Let $P=\left\{\bar{x}_{1}, \ldots, \bar{x}_{k}\right\}$ denote a set of points in $\mathbb{R}^{2}$, and $V_{P}=$ $\left\{\mathcal{R}_{i} ; 1 \leq i \leq n\right\}$ its Voronoi diagram in the plane. We call maximal empty circle centered in a point $\bar{p}$ to $D\left(\bar{p}, r_{\bar{p}}\right)=\left\{\bar{x} \in \mathbb{R}^{2}: d(\bar{x}, \bar{p}) \leq r_{\bar{p}}\right\}$ where $r_{\bar{p}}=$ $\min \left\{d\left(\bar{p}, \bar{x}_{i}\right) ; \bar{x}_{i} \in P, \bar{x}_{i} \neq \bar{p}\right\}$.

Proposition 4. Let $P=\left\{\bar{x}_{1}, \ldots, \bar{x}_{k}\right\} \subset \mathbb{R}^{2}$, and $V_{P}=\left\{\mathcal{R}_{i} ; 1 \leq i \leq n\right\}$ its Voronoi diagram in the plane. The following properties hold true:

i) A point $\bar{q} \in \mathbb{R}^{2}$ is a vertex of the Voronoi diagram $V_{P}$ if and only if $\left|D\left(\bar{q}, r_{\bar{q}}\right) \cap P\right| \geq 3$. 
ii) Given $1 \leq i \neq j \leq k$, the bisector of the segment that joins $\bar{x}_{i}$ to $\bar{x}_{j}$ is a boundary of $V_{P}$ if and only there exist a point $\bar{q}$ in that bisector such that $\left|D\left(\bar{q}, r_{\bar{q}}\right) \cap P\right|=2$.

Proof: This directly follows from the fact $\mathcal{R}_{i} \cap \mathcal{R}_{j} \subset\left\{\bar{x} \in \mathbb{R}^{2}: d\left(\bar{x}, \bar{x}_{i}\right)=\right.$ $\left.d\left(\bar{x}, \bar{x}_{j}\right)\right\}$. (See also Theorem 7.4 in [9] for further results in this direction).

Remark 11. About how to compute the Voronoi diagram of a set of points $P=\left\{\bar{x}_{1}, \ldots, \bar{x}_{k}\right\}$ in the plane $\mathbb{R}^{2}$, it is relevant to know that there are many algorithms that calculate it (see e.g., the so-called "Fortune algorithm", introduced in [28]). There is also a recursive algorithm that calculates $V_{P \cup\left\{\bar{x}_{n+1}\right\}}$ from $V_{P}$ with a complexity of $O\left(n^{2}\right)$. This is important because, in a sense, this follows the spirit of Definition 5 about reducible operators. Furthermore, there are more efficient algorithms that compute $V_{P}$ with a complexity $O(n \log n)$, as proved in Section 7.2 in [9] (see also [38]).

The Voronoi diagram $V_{P}$ of a set of points $P=\left\{\bar{x}_{1}, \ldots, \bar{x}_{k}\right\}$ in the plane $\mathbb{R}^{2}$ is dual to the Delaunay triangulation that the set $P$ induces in the lane (see Definition 16 below). Any of them can be got from the other one by means of a suitable $O(n)$ time algorithm (see $[38,9]$ ).

Definition 15. Let $P=\left\{\bar{x}_{1}, \ldots, \bar{x}_{k}\right\}$ be a non collinear set of points in the plane. A triangulation is a partition of the convex hull of $P$ into triangles in a way such that the following conditions hold:

i) Each point in $P$ is a vertex of some triangle in the partition.

ii) Each vertex of a triangle in the partition is a point of the set $P$.

iii) No edge connecting two vertices in the partition can be added to the partition without intersecting one of already existing edges.

Definition 16. We will say that a triangulation satisfies the Delaunay condition (or, equivalently, we say that it is a Delaunay triangulation) if the circumcircle of every triangle in the corresponding partition that defines that triangulation does not contain any other different vertex of a triangle in such partition (see $[23])$.

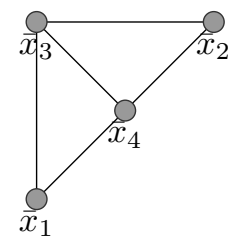

Proposition 5. Given three non collinear points $A=\left(a_{1}, a_{2}\right), B=\left(b_{1}, b_{2}\right)$, $C=\left(c_{1}, c_{2}\right)$ in the real plane, a point $D=\left(d_{1}, d_{2}\right) \in \mathbb{R}^{2}$ lies inside the circum- 
circle of the triangle of vertices $A, B$ and $C$ if and only if

$$
\left|\begin{array}{llll}
a_{1} & a_{2} & a_{1}^{2}+a_{2}^{2} & 1 \\
b_{1} & b_{2} & b_{1}^{2}+b_{2}^{2} & 1 \\
c_{1} & c_{2} & c_{1}^{2}+c_{2}^{2} & 1 \\
d_{1} & d_{2} & d_{1}^{2}+d_{2}^{2} & 1
\end{array}\right|=\left|\begin{array}{ccc}
a_{1}-d_{1} & a_{2}-d_{2} & \left(a_{1}-d_{1}\right)^{2}+\left(a_{2}-d_{2}\right)^{2} \\
b_{1}-d_{1} & b_{2}-d_{2} & \left(b_{1}-d_{1}\right)^{2}+\left(b_{2}-d_{2}\right)^{2} \\
c_{1}-d_{1} & c_{2}-d_{2} & \left(c_{1}-d_{1}\right)^{2}+\left(c_{2}-d_{2}\right)^{2}
\end{array}\right|>0 .
$$

Proof: See for instance [43].

Definition 17. Consider a triangulation of a set of points $P=\left\{\bar{x}_{1}, \ldots, \bar{x}_{k}\right\}$ in the plane $\mathbb{R}^{2}$. Suppose that it has $m$ triangles. We pay attention to the $3 m$ angles that come from all those triangles in the partition, sorting those angles by increasing value, say $\alpha_{1} \leq \ldots \leq \alpha_{3 m}$. The vector $\left(\alpha_{1}, \ldots, \alpha_{3 m}\right)$ is said to be the angle-vector of the given partition of $P$. Among all the partitions of $P$ we consider all their corresponding angle-vectors. We endow the resulting set of angle-vectors with the lexicographic order. A triangulation that gives rise to the maximal angle-vector in that lexicographic linear order is said to be angle-optimal.

Proposition 6. Any angle-optimal triangulation of $P$ is a Delaunay triangulation of $P$. Moreover, any Delaunay triangulation of $P$ maximizes the minimum angle over all triangulations of $P$.

Proof: See Theorem 9.9. in [9]. See also [27] for further details.

Finally we include a few words about the use of Voronoi diagrams in aggregation of finite fuzzy sets. In fact, in this case they are more used to do selections, that is aggregations in which the resulting vector of the aggregation is one of the points in the original cloud. Depending on the contexts analyzed, sometimes this result will be the point whose corresponding Voronoi cell has the smallest area. But also, in other different approaches, the selected point will be the one with the biggest Voronoi cell.

\subsection{Distances to a cloud. A geometrical approach to avoid outliers}

Assume that we are given a finite set of points (vectors) in an Euclidean space. That set of points is usually called a cloud. Each vector in the cloud represents different measurements or data of a single natural phenomenon or distinct "objective" opinions. Since there is only one phenomenon, we may expect a priori that the points are close one another.

It is usual that an error is associated to each measurement (due to noise, biases, etc.). This error can be represented by a ball around the corresponding point, so that the "actual value" of the phenomenon should be inside that interval or ball, not exceeding a margin represented by the radius of the ball.

Furthermore, some of those measurements could appear distorted by punctual and unpredictable phenomena outside the experimental frame. This will give rise to strange points (usually known as the outliers). In general, the researchers that are analyzing the phenomenon know well, even at first sight, 
that those outliers must be discarded. They are not significant in the problem studied. And, what is even worse, they jeopardize the exact measurements and data.

The outliers problem is a recurrent problem in scientific research (see e.g. [2]), and various statistical methods have been implemented to avoid their damaging effect in data treatment (see e.g. [8, 42]).

Bearing in mind these previous ideas coming from empirical research, aggregation points in a cloud should have as more data points as possible in their neighboring balls, even when the diameter of them is as small as we please.

Inspired in this intuition, we introduce a new aggregation procedure.

Let $X=\left\{\bar{x}_{1}, \ldots, \bar{x}_{k}\right\} \subset[0,1]^{n}$ denote a cloud (set of points in the $n$ dimensional unit cube). Given $\bar{p} \in[0,1]^{n}$ and $r>0$, the ball of center $\bar{p}$ and radius $r$ is denoted by $B(\bar{p}, r)=\left\{\bar{x} \in[0,1]^{n}:|| \bar{p}-\bar{x}||<r\right\}$. Let $|B(\bar{p}, r) \cap X|$ stand for the cardinality of the subset of points of the cloud $X$ that belong to the ball $B(\bar{p}, r)$.

Let us also consider a continuous function $f:(0,+\infty) \rightarrow(0,+\infty)$ satisfying the following conditions:

i) $\lim _{t \rightarrow 0} f(t)=+\infty=\lim _{t \rightarrow+\infty} f(t)$.

ii) There exists $t_{0}>0$ such that $f$ is decreasing in $\left(0, t_{0}\right)$ and increasing in $\left(t_{0},+\infty\right)$.

Now, let us define a function $\phi_{X}:[0,1]^{n} \rightarrow \mathbb{R}$ representing how distant is a point $p$ to the cloud $X$ by setting the smallest radius $r$ such maximizes $\frac{|\overline{B(p, r)} \cap X|}{f(r)}$, as follows: Fixed $p$, first we compute

$$
M(p)=\max _{r>0}\left\{\frac{|\overline{B(p, r)} \cap X|}{f(r)}\right\} .
$$

It may happen that this maximum $M$ is attained for more than one radius $r$. Therefore, we take then the infimum of all the radius $r$ such that $M(p)=$ $\frac{|\overline{B(p, r)} \cap X|}{f(r)}$. Denote that infimum by $r(p)$. Thus we finally declare that: $\phi_{X}(p)=$ $r(p)$.

Remark 12. The function $f$ will be chosen according to the specific problem we are dealing with. Restrictions over $f$ are necessary in order to punish vectors that lie far from almost every point of $X$ (here $r$ would be too big), as well as the ones that are quite close to a single point of $X$, but far from all the others (here $r$ would be too small). The number $t_{0}$ is a threshold to measure the aforementioned "closedness". Thus, $\phi_{X}$ captures interesting ideas not usually contemplated in traditional aggregation functions. Here, we try to pay attention to an intuition in which the best candidates $p \in[0,1]^{n}$ to be selected as aggregation points are the ones which have more points in a small neighborhood, i.e. 
the ones with the smallest $\phi_{X}(p)=r(p)$. However, it may still happen that the minimum is not unique. Anyway, this gives us an idea about how to quantify the closedness to the point $p$ of points in the cloud $X$, trying to measure how the point $p$ is "surrounded" by the points in $X$.

\section{Notice that:}

a) $\phi_{X}$ ignores points of $X$ which are far from the the majority of the elements in $X$. We can considerate those bad points as outliers. In comparison to other aggregation procedures, we may observe that $O W A$ operators only diminish the contribution of outliers (assigning them low weights), but they do not rule out them unless their assigned weights are zero.

b) Unfortunately, a new trouble can appear with this new aggregation procedure if $X$ has more than just one single tendency (e.g. $X$ consist of two separated clouds of points, one cloud located quite far from the other one). In that situation, the candidates proposed by $\phi_{X}$ may fail to be "intermediate points" located between those independent clouds.

Example 2. Consider the cloud of five points $X=\{(0.1,0.1),(0.18,0.2),(0.19$, $0.14),(0.15,0.12),(0.4,0.1)\}$ in the unit plane $[0,1]^{2}$. Let $Y=X \backslash\{(0.4,0.1)\}$.
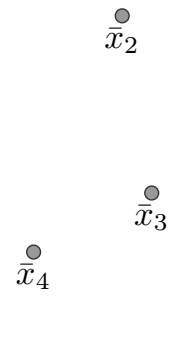

\section{$\stackrel{\circ}{x}_{5}$}

Take the function $f:(0,+\infty) \rightarrow(0,+\infty)$, defined as follows:

$$
f(r)=-\ln \left(r^{\frac{1}{10}}\right)-10 \cdot r \cdot \ln \left(1-\frac{r}{\sqrt{2}}\right) .
$$

It is plain that $(0.4,0.1)$ in $X$ is quite far from all the other ones (that belong to $Y)$. Hence, we understand $Y$ as a cloud and $\bar{x}_{5}=(0.4,0.1)$ as an outlier. A few computations prove that $(0.4,0.1)$ has no effect on the final aggregation made by this new procedure. The aggregation procedure leads to the point $(0.14,0.15)$ no matter if we work with the original cloud $X$ or we ignore the point $x_{5}$ and work with $Y$. Moreover, some intermediate calculation of $\phi_{X}$ and $\phi_{Y}$ yield the same result in some selected points. For instance: $\phi_{X}(0.1,0,1)=\phi_{Y}(0.1,0.1)=$ 0.251 , whereas $\phi_{X}(0.4,0,1)=\phi_{Y}(0.4,0.1)=0.064$. Furthermore, if we compute first the arithmetic mean of the points in $X$ (respectively, in $Y$ ), and we denote it by $\bar{x}$ (respectively, by $\bar{y}$ ), we also have here that $\phi_{X}(\bar{x})=\phi_{Y}(\bar{x})=0.108$, and $\phi_{X}(\bar{y})=\phi_{Y}(\bar{y})=0.068$. 


\subsection{A new aggregation method inspired on an asset allocation model}

We could consider another geometrical method inspired in the non-parametric asset allocation model denoted Entropy Pooling (EP) introduced by A. Meucci in 2008 (see [35]). This method combines an arbitrary market model with completely general views on this market, in order to produce a posterior distribution (see also [10] for previous studies on similar items). To do so, in EP the views are interpreted as statements that deform the prior distribution so that the minimum of unnecessary structure is imposed, measuring this discrepancy through an entropy function. If there are two or more investors expressing views, with different levels of confidence, we can aggregate all of them using the opinion pooling technique.

Based on this idea, we could reinterpret it from a geometrical point of view. This will give rise to another aggregation method for fuzzy sets on a finite universe. As mentioned in the Introduction, fuzzy sets of these kind can be considered as vectors in the $n$-dimensional unit cube, and so they can play the role of vectors of probabilities representing the market model. Considering the investors views, which can be easily translated into geometrical conditions in the unit cube, we could combine them through the entropy pooling technique in order to obtain a new vector in the $n$-dimensional unit cube, that can consequently be interpreted as a new finite fuzzy set.

Bearing this idea in mind, we could reinterpret it from a geometrical point of view giving us an aggregation method for fuzzy sets on a finite universe.

To introduce and describe the method, we are going to consider first the case inspired in one investor expressing views. Then we will analyze the case in which two or more investors are involved.

Base case: Only one investor

Let $U=\left\{u_{1}, \ldots, u_{n}\right\}$ be a finite universe. Let $\mathcal{F}$ stand for the family of fuzzy sets of $U$. Each fuzzy set $X \in \mathcal{F}$ can be interpreted as a vector in the $n$-dimensional unit cube $[0,1]^{n}$. Thus, it can also play the role of a vector of probabilities $\mathbf{p} \in[0,1]^{n}$. Those probabilities would correspond to joint scenarios representing the market model.

In the EP technique, in order to represent the posterior distribution of the market that includes the views, instead of generating new simulations, the same scenarios are used, but with a different vector of probabilities $\tilde{\mathbf{p}}$. The general views can be written as a set of linear constraints on the new, yet to be determined, probabilities:

$$
\underline{\mathbf{a}} \leq^{*} \tilde{\mathbf{p}} \mathbf{A} \leq^{*} \overline{\mathbf{a}}
$$

where $\underline{\mathbf{a}}$ and $\overline{\mathbf{a}}$ are constant vectors in $\mathbb{R}^{n}$, and $\mathbf{A}$ is a constant $n \times n$ matrix of real numbers. (Here " $\leq$ " stands for the partial order in $\mathbb{R}^{n}$ so that given $\bar{x}=\left(x_{1}, \ldots x_{n}\right), \bar{y}=\left(y_{1}, \ldots, y_{n}\right) \in \mathbb{R}^{n}$ we declare that $\bar{x} \leq^{*} \bar{y}$ provided that $x_{i} \leq y_{i}$ holds true for every $1 \leq i \leq n$.)

Inspired in this idea, from a geometrical point of view, we could consider not only this kind of views, but also we could generalize it through a subset $\mathcal{C} \subseteq[0,1]^{n}$, so that $\tilde{\mathbf{p}} \in C$. 
The relative entropy, in its discrete version, between $\mathbf{p}$ and $\tilde{\mathbf{p}}$ can be measured as:

$$
\mathcal{E}(\mathbf{p}, \tilde{\mathbf{p}}) \equiv \sum_{j=1}^{n} \tilde{p}_{j}\left[\ln \left(\tilde{p}_{j}\right)-\ln \left(p_{j}\right)\right]
$$

We can define now the following modifier $F: \mathcal{F} \rightarrow \mathcal{F}$ as $F(\mathbf{p})=\mathbf{p}^{\prime}$ such that

$$
\mathcal{E}\left(\mathbf{p}, \mathbf{p}^{\prime}\right)=\min \{\mathcal{E}(\mathbf{p}, \tilde{\mathbf{p}}) \mid \tilde{\mathbf{p}} \in \mathcal{C}\} .
$$

There is a last step which allows as to define a new modifier $G: \mathcal{F} \rightarrow \mathcal{F}$ considering the pooling parameter $c \in[0,1]$ which represents the confidence level in the views:

$$
G(\mathbf{p}) \equiv(1-c) \mathbf{p}+c F(\mathbf{p}) .
$$

General case: Multi investor context

Inspired in the case in which Entropy Pooling is used in a multi-manager context, to aggregate their separate views considering their confidence-weighted average, we are going to translate it into our model.

Let $\mathcal{C}_{i} \subseteq[0,1]^{n} \quad(i=1, \ldots, k)$. We define the following family of modifiers $F_{i}: \mathcal{F} \rightarrow \mathcal{F}(i=1, \ldots, k)$ as $F_{i}(\mathbf{p})=\mathbf{p}_{i}^{\prime}$ such that

$$
\mathcal{E}\left(\mathbf{p}, \mathbf{p}_{i}^{\prime}\right)=\min \left\{\mathcal{E}(\mathbf{p}, \tilde{\mathbf{p}}) \mid \tilde{\mathbf{p}} \in \mathcal{C}_{i}\right\} .
$$

We can define now the aggregation operator

$$
G(\mathbf{p})=\sum_{i=1}^{k} c_{i} F_{i}(\mathbf{p})\left(c_{i} \in[0,1], \sum_{i=1}^{k} c_{i}=1\right) .
$$

Remark 13. We could consider a generalization in which the role of entropy could be played by any distance function.

\section{Multidisciplinary applications}

All the previous ideas and aggregation methods have a wide scope in miscellaneous applications. For instance, in [26] we find references to eighteen different applications of Voronoi diagrams and Delaunay triangulations. Here are some of them:

1) Anthropology and Archaeology: Identify the parts of a region under the influence of different neolithic clans, chiefdoms, ceremonial centers, or hill forts.

2) Astronomy: Identify clusters of stars and clusters of galaxies.

3) Biology, Ecology, Forestry: Model and analyze plant competition through the "area potentially available to a tree".

4) Cartography: Place together satellite photographs into large "mosaic maps". 
5) Crystallography and Chemistry: Study chemical properties of metallic sodium by means of the so-called "Wigner-Seitz regions".

$\cdots$

17) Statistics and Data Analysis: Analyze statistical clustering through "natural neighbors interpolation".

18) Zoology: Model and analyze the territories of animals.

In the present section we comment some other possible applications of several aggregations methods previously considered in the manuscript.

\subsection{Image Processing}

Perhaps a framework in which the applications of aggregation operators as well as modifiers is more evident is that of Image Processing. As commented before, a black and white picture can be understood as a function from a finite universe of pixels into the unit interval $[0,1]$. In addition, a full colors picture can be interpreted through three functions from that finite university of pixels into $[0,1]$, namely one function for each primary color (a RGB system). Any kind of photo retouch can then be framed through suitable operators or modifiers on those functions. At this stage, it is relevant to bear in mind the classical modifiers introduced by V. Novák as linguistic modifiers in contexts of fuzzy logic (see [36], Ch.3, pp. 104-117). The effect of the so called "concentration", "dilation" or "intensification" modifiers (among others) correspond to photo retouches in which a color is blurred or highlighted at convenience. These modifiers directly depend on suitable functions from $[0,1]$ into itself whose effect is, say, to increase or decrease a given number in $[0,1]$. For instance, the function $f:[0,1] \rightarrow[0,1]$ given by $f(t)=t^{2}$, known as concentration in Novák's setting ([36]) provokes that a color is blurred because $x^{2}<x$ if $x \in(0,1)$. In fact, any function $g:[0,1] \rightarrow[0,1]$ such that $g(t)<t$ if $t \in(0,1)$ would have an effect of blurring colors. Also, any function $h:[0,1] \rightarrow[0,1]$ such that $h(t)>t$ if $t \in(0,1)$ would have the opposite effect, that is, it would highlight colors. Other more sophisticated functions with double effect of blurring some pixels whereas highlighting others can also be used here, built in terms of suitable analytical functions from the unit interval into itself.

Another common task in image processing is the reduction of an image by transforming it in another one with a lower number of rows and columns in its spatial resolution as faithfully as possible. The technique consists in dividing the original image in blocks of pixels. Then, an aggregation operator is applied to the sets of pixels in each block in order to get a single pixel which preserves the properties of the original block as much as possible. If a weighted averaging is used for this aggregation, the weight assigned to each pixel depends only on its position. However, if we choose an OWA operator for each aggregation, then the weight assigned to each pixel will depend only on its value, i. e., on the place that each value gets when all of the values of the same block are non-increasingly rearranged in a descending chain. Working this way, we can decide, for instance, 
to assign the highest weights to the highest values in order to darken the image. Contrariwise, we could give the highest weights to the lowest values in order to lighten the image.

\subsection{Networks}

Fermat points constitute an important device in the study of networks and systems of communication (e.g.: transmitters and receptors). Suppose for instance that $n$ points in the plane represent $n$ different cities, so that we want to find a point $F$ in order to locate there a transmitter (e.g. radio). If the $n$ points were cocyclic, quite probably we would select the center of the circle to which those points belong, because the distances between the center and each point is the same, and consequently the signal reaches each of the points at the same time. But, when the points do not lie in a circle, it is natural to choose the Fermat point, because the total energy, understood as the sum of distances or routes operated by the signal is kept to a minimum. This is also a typical problem in facility location, when working with models that try to locate a facility to minimize costs of transportation.

\subsection{Bets}

Assume that you want to bet money on the result of a football match. In any betting house you will find the amount that they will pay you, per unit of money invested, depending on the final result. For instance $(1.6,2.8,7)$ means that the betting house will pay you, per dollar invested, 1.6 dollars if the local team wins, 2.8 dollars if the match ends in a tie, and 7 dollars if the visitor team is the winner. Perhaps, in order to better invest your money, you visit not only one, but several different betting houses. Each of them has an estimation of the money they will pay you, but the corresponding payments may vary from one betting house to another one, because their estimations may fail to coincide. Thus, if you have at hand the vectors of payments (say, for instance: $(1.6,2.8,7) ;(1,61,2.75,8) ;(1.4,3,8.5))$ you could try to find some distribution of your money assigning weights to the different betting houses in the way you like (e.g.: you may decide to invest $60 \%$ of your money in the former house, $30 \%$ in the second one and $10 \%$ in the third one). This leads to an average vector of payments that, in this case, comes from a weighted arithmetic mean. More sophisticated systems would try to find a distribution of money such that the resulting average vector of payments coincides, say, with the Fermat point. In addition, from a vector of payments one could compute the vector of probabilities ${ }^{5}$ that the house assigns to each result of the match (see e.g. [45]). of These vectors of probabilities lie in the unit cube $[0,1]^{3}$ so that they can also be considered as fuzzy sets on a universe of three elements.

\footnotetext{
${ }^{5}$ This should be the vector of inverses of payments, but sometimes the sum of these inverses is not 1 . This is due to the taxes, the money that the betting house gains, and sometimes, some bonus to attract the gamblers. For instance, in the vector of payments $(1.6,2.8,7)$, the $\operatorname{sum} \frac{1}{1.6}+\frac{1}{2.8}+\frac{1}{7}=1.125>1$.
} 
Thus, they can be aggregated in a suitable way, so that you can also have an idea about which is the estimated probability of each result of the match, as an average of the estimated probabilities assigned a priori by the betting houses.

\subsection{Sports}

Voronoi diagrams have applications in sports, in a wide sense (see [45]). For instance, in a collective game as, say, handball, it is interesting to look for the players who dominate a wide part of the playground. To this extent we may think that the player whose corresponding Voronoi cell has the biggest area is the best. Or maybe we may think differently, so that the most clever players are those that force more people to be around and close to them. In that interpretation, we would pay attention to the player whose Voronoi cell is the smallest one. A wide analysis of situations of this sort, explained in a didactical and funny manner, may be seen in [45].

\section{Conclusion}

Aggregation procedures based on some geometrical idea could also be an interesting tool to handle fuzzy sets defined on a finite universe, so interpreting the fuzzy sets as points in a unit cube. The possibilities that these alternative methods suggest for application in multidisciplinary fields tell us that they deserve attention and should never be put apart from other typical aggregation methods based on, say, algebraic or analytical settings. Among the geometrical procedures, some of them are related to classical problems in Computational Geometry, as, e.g., the computation of Fermat and Weber points. Moreover, some classical aggregation operators, not usually considered as "geometrical", can also be given some enlightening geometrical interpretation, as it is the case of the OWA operators.

\section{Acknowledgements}

This work has been partially supported by the research projects TIN201347605-P, ECO2015-65031-R, MTM2015-63608-P (MINECO/ AEI-FEDER, UE), TIN2016-77356-P (MINECO/ AEI-FEDER, UE), TIN2016-81731-REDT (LODISCO II) (MINECO/ AEI-FEDER, UE) and the Research Services of the Public University of Navarre (Spain).

Thanks are also given to the organizers and participants in the Joint 17th World Congress of International Fuzzy Systems Association and 9th International Conference on Soft Computing and Intelligent Systems (IFSA-SCIS 2017), held in Otsu, Japan (June 27th-30th 2017), for their valuable suggestions and comments. Some of the ideas introduced in the present manuscript were communicated there in a special session devoted to "Aggregation functions: theory and practice". 


\section{References}

[1] S. Abu-Saymeh and M. Hajja (1997), On the Fermat-Torricelli Points of Tetrahedra and of Higher Dimensional Simplexes, Mathematics Magazine 70 (5), 372-378.

[2] H. Aguinis, R.K. Gottfredson and H. Joo (2013), Best-practice recommendations for defining, identifying and handling outliers, Organizational Research Methods 16 (2), 270-301.

[3] C. Alsina, M. J. Frank and B. Schweizer (2006), Associative Functions. Triangular Norms and Copulas, New Jersey: Word Scientific.

[4] G. Aumann (1943), Über Raüme mit Mittelbindungen, Math. Annalen 119, 210-215.

[5] G. Beliakov (2010), Optimization and aggregation functions, in W.A. Lodwick and J. Kacprzyk (eds.): Fuzzy Optimization, STUDFUZZ 254, pp. 77-108, Berlin-Heidelberg: Springer.

[6] G. Beliakov, H. Bustince, S. James, T. Calvo and J. Fernandez (2012), Aggregation for Atanassov's intuitionistic and interval valued fuzzy sets: the median operator, IEEE Transactions on Fuzzy Systems 20 (3), 487498.

[7] R. Bellman, R. Kalaba and L.A. Zadeh (1966), Abstraction and pattern classification, J. Math. Anal. Appl. 13, 1-7.

[8] I. Ben-Gal (2005), Outlier Detection, in: O. Maimon O and L. Rokach (eds.): Data Mining and Knowledge Discovery Handbook, Chapter 7, pp. 131-146, Boston, MA: Springer.

[9] M. de Berg, O. Cheong, M. van Kreveld and M. Overmars (2008), Computational Geometry. Algorithms and Applications (Third Edition), Berlin: Springer-Verlag.

[10] F. Black and R. Litterman (1990), Asset Allocation: Combining Investor Views with Market Equilibrium, Fixed Income Research, New York: Goldman, Sachs and Company.

[11] C.J. Bradley and G.C. Smith (2006), The locations of triangle centers, Forum Geometricorum 6, 57-70.

[12] H. Bustince, M.J. Campión, F.J. Fernández, E. Induráin, and M.D. Ugarte (2014), New trends on the permutability equation, Aequat. Math. 88, 211232.

[13] M.J. Campión, J.C. Candeal, R.G. Catalán, J.R. De Miguel, E. Induráin and J.A. Molina (2011), Aggregation of preferences in crisp and fuzzy settings: functional equations leading to possibility results, Internat. J. Uncertain. Fuzziness Knowledge-Based Syst. 19(1), 89-114. 
[14] J.C. Candeal, J.R. De Miguel, E. Induráin and E. Olóriz (1997), Associativity equation revisited, Pub. Math. Debrecen 51 (1-2), 133-144.

[15] J.C. Candeal and E. Induráin (1994), Medias generalizadas y aplicaciones, Extracta Math. 9 (3), 139-159.

[16] J.C. Candeal and E. Induráin (2017), Point-sensitive aggregation operators: functional equations and applications to Social Choice, International Journal of Uncertainty, Fuzziness and Knowledge-Based Systems 25 (6), 973-986.

[17] R. Chandrasekaran, A. Tamir (1989), Open questions concerning Weiszfeld's algorithm for the Fermat-Weber location problem, Mathematical Programming 44 (1-3), 293-295.

[18] G. Chichilnisky and G.M. Heal (1983), Necessary and sufficient conditions for a resolution of the social choice paradox, J. Econom. Theory 31(1), $68-87$.

[19] L. Cooper (1963), Location-allocation problems, Operations Research 11(3), 331-343.

[20] H.S.M. Coxeter (1989), Introduction to Geometry (Second Edition), New York: Wiley.

[21] R. Craigen and Z. Páles (1989), The associativity equation revisited. Aequat. Math. 37, 306-312 .

[22] L. De Miguel, M.J. Campión, J.C. Candeal, E. Induráin and D. Paternain (2017), Pointwise aggregation of maps: Its structural functional equation and some applications to social choice theory, Fuzzy Sets and Systems 325, $137-151$.

[23] B. Delaunay (1934), Sur la sphére vide, Bulletin de l'Académie des Sciences de l' URSS. Classe des sciences mathématiques et naturelles 6, 793-800.

[24] R. Descartes (1644), Principia Philosophiae, Amsterdam: Louis Elzevir.

[25] P.G.L. Dirichlet (1850), Über die Reduktion der positiven quadratischen Formen mit drei unbestimmten ganzen Zahlen, Journal für die Reine und Angewandte Mathematik 40, 209-227.

[26] R.L.S. Drysdale (1993) Voronoi Diagrams: Applications from Archaeology to Zoology, Geometry in Action (July 19, 1993), also at http://mathforum.org/mathed/dimacs/lecture.4.html or http://www.ics.uci.edu/ eppstein/gina/scot.drysdale.html.

[27] H. Edelsbrunner, T.S. Tang, R. Waupotitsch (1992) An $O\left(n^{2} \operatorname{logn}\right)$ time algorithm for the minimax angle triangulation, SIAM Journal on Scientific and Statistical Computing 13 (4), 994-1008. 
[28] S. Fortune (1986), A sweepline algorithm for Voronoi diagrams. Proceedings of the second annual symposium on Computational geometry, New York: Yorktown Heights, pp.313-322.

[29] P. Hájek and R. Mesiar (2008), On copulas, quasicopulas and fuzzy logic, Soft Comput. 12, 1239-1243.

[30] M. Hajja (1994), An advanced calculus approach to finding the Fermat point, Mathematics Magazine 67, 29-34.

[31] E.P. Klement, R. Mesiar and E. Pap (2000), Triangular Norms, Dordrecht: Kluwer. ISBN 0-7923-6416-3.

[32] L.L. Lindelöf (1867), Sur les maxima et minima d'une fonction des rayons vecteurs menés d'un point mobile à plusieurs centres fixes, Acta Soc. Sc. Fenn. 8, 191-203.

[33] J.-L. Marichal (2000), On the associativity functional equation, Fuzzy Sets and Systems 114(3), 381-389.

[34] J. Martín and G. Mayor (2011), Multi-argument distances, Fuzzy Sets and Systems 167, 92-100.

[35] A. Meucci, (2008), Fully flexible views: theory and practice, Risk 21 (10), 97-102.

[36] V. Novák (1989), Fuzzy sets and their applications, Bristol and Philadelphia: Adam Hilger. ISBN 0-85274-583-4.

[37] G. Ochoa, I. Lizasoáin, D. Paternain, H. Bustince and N.R. Pal (2017), From quantitative to qualitative orness for lattice OWA operators, International Journal of General Systems, 46(6), 640-669.

[38] A. Okabe, B. Boots, K. Sugihara and S.N. Chiu (2000), Spatial Tessellations - Concepts and Applications of Voronoi Diagrams (2nd edition), New York: John Wiley.

[39] O.L. Palacios-Vélez, F. Pedraza-Oropeza and B.S. Escobar-Villagrán (2015), An algebraic approach to finding the Fermat-Torricelli point, International Journal of Mathematical Education in Science and Technology $46(8), 1252-1259$.

[40] F. Plastria (2006), Four-point Fermat location problems revisited. New proofs and extensions of old results, IMA Journal of Management Mathematics 17 (4), 387-396.

[41] J.J. Risler (1992), Mathematical Methods for CAD (first edition), Paris: Masson.

[42] P.J. Rousseuw and M. Hubert (2011), Robust statistics for outlier detection, WIREs Data Mining Knowl. Discov. 1(1) , 73-79. 
[43] J.R. Shewchuk (1997), Adaptive precision floating-point arithmetic and fast robust geometric predicates, Discrete and Computational Geometry 18, 305-363.

[44] A. Stupn̆anová and A. Kolesárová (2011), Associative n-dimensional copulas, Kybernetika 47 (1), 93-99.

[45] D. Sumpter (2016) Soccermatics. Mathematical Adventures in the Beautiful Game, London, New York, Sydney and New Delhi: Bloomsbury Sigma.

[46] A.H. Thiessen (1911) Precipitation averages for large areas, Monthly Weather Review 39(7), 1082-1084.

[47] Y. Vardi and C.-H. Zhang (2000), The multivariate $L_{1}$-median and associated data depth, Proceedings of the National Academy of Sciences of the United States of America 97 (4), 1423-1426.

[48] G.F. Voronoi (1907) Nouvelles applications des paramètres continus à la théorie des formes quadratiques I. Sur quelques propriétés des formes quadratiques positives parfaites, Journal für die Reine und Angewandte Mathematik 133, 97-178.

[49] G.F. Voronoi (1908) Nouvelles applications des paramètres continus à la théorie des formes quadratiques II. Recherches sur les paralléloèdres primitifs, Journal für die Reine und Angewandte Mathematik 134, 198-287.

[50] A. Weber (1909), Über den Standort der Industrien, Tübingen. (Translated by C.J. Friederich (1929) as "Theory of the location of industries", Chicago: University of Chicago Press).

[51] E. Weiszfeld (1937), Sur le point par lequel la somme des distances de $n$ points donnés est minimum, Tohoku Mathematics Journal 43, 355-386.

[52] G.O. Wesolowsky (1993), The Weber problem: history and perspectives, Location Science 1(1), 5-23.

[53] R.R. Yager (1988), On ordered weighted averaging aggregation operators in multi-criteria decision making, IEEE Transactions on Systems, Man and Cybernetics 18, 183-190.

[54] L.A. Zadeh (1965), Fuzzy Sets, Information and Control 8, 338-353. 